\title{
Spectrum and genus of commuting graphs of some classes of finite rings
}

\author{
Jutirekha Dutta, Walaa Nabil Taha Fasfous, \\ AND RAJAT KANTI NATH
}

\begin{abstract}
We consider commuting graphs of some classes of finite rings and compute their spectrum and genus. We show that the commuting graph of a finite CC-ring is integral. We also characterize some finite rings whose commuting graphs are planar.
\end{abstract}

\section{Introduction}

Let $R$ be a finite non-commutative ring and let $Z(R)$ be the center of $R$. Let $\Gamma_{R}$ be the commuting graph of $R$. Then $\Gamma_{R}$ is an undirected graph with vertex set $R \backslash Z(R)$, and two distinct vertices $a, b$ are adjacent if $a b=b a$. In $[1,4,5,13,17,18,19]$, various graph theoretic aspects of $\Gamma_{R}$ have been studied for different families of finite rings. Some generalizations of $\Gamma_{R}$ are also considered in $[3,8]$. In this paper, we compute the spectrum and genus of $\Gamma_{R}$ for some classes of finite rings. We show that $\Gamma_{R}$ is integral if $R$ is a finite CC-ring. We also characterize some finite rings whose commuting graphs are planar.

Let $\operatorname{Spec}(\mathcal{G})$ denote the spectrum of a graph $\mathcal{G}$. Then $\operatorname{Spec}(\mathcal{G}):=\left\{\lambda_{1}^{k_{1}}, \lambda_{2}^{k_{2}}\right.$, $\left.\ldots, \lambda_{n}^{k_{n}}\right\}$, where $\lambda_{1}, \lambda_{2}, \ldots, \lambda_{n}$ are the eigenvalues of the adjacency matrix of $\mathcal{G}$ with multiplicities $k_{1}, k_{2}, \ldots, k_{n}$, respectively. If $\operatorname{Spec}(\mathcal{G})$ contains only integers then $\mathcal{G}$ is called an integral graph. In 1974, Harary and Schwenk [14] introduced the notion of an integral graph. Following them a number of mathematicians have considered this class of graphs in their studies (see, for example, $[2,15,20])$. In $[11,12]$, Dutta and Nath have determined several groups whose commuting graphs are integral.

Received April 5, 2017.

2010 Mathematics Subject Classification. 16P10; 05C25; 05C50.

Key words and phrases. Integral graph; commuting graph; spectrum; genus.

http://dx.doi.org/10.12697/ACUTM.2019.23.01

Corresponding author: Rajat Kanti Nath 
Let $K_{n}$ be the complete graph on $n$ vertices. It is well known that $\operatorname{Spec}\left(K_{n}\right)=\left\{(-1)^{n-1},(n-1)^{1}\right\}$ and hence $K_{n}$ is integral. Further, if $\mathcal{G}=\bigcup_{i=1}^{m} K_{n_{i}}$ then we have

$$
\operatorname{Spec}(\mathcal{G})=\left\{(-1)^{\sum_{i=1}^{m} n_{i}-m},\left(n_{1}-1\right)^{1},\left(n_{2}-1\right)^{1}, \ldots,\left(n_{m}-1\right)^{1}\right\} .
$$

The smallest non-negative integer $n$ such that a graph $\mathcal{G}$ can be embedded on the surface obtained by attaching $n$ handles to a sphere is called the genus of $\mathcal{G}$. We write $\gamma(\mathcal{G})$ to denote the genus of a graph $\mathcal{G}$. It is worth mentioning that (see [21, Theorem 6-38])

$$
\gamma\left(K_{n}\right)=\left\lceil\frac{(n-3)(n-4)}{12}\right\rceil \text { if } n \geq 3 .
$$

Also, if $\mathcal{G}=\bigcup_{i=1}^{m} K_{n_{i}}$ then by [6, Corollary 2] we have

$$
\gamma(\mathcal{G})=\sum_{i=1}^{m} \gamma\left(K_{n_{i}}\right)
$$

A graph $\mathcal{G}$ is called planar or toroidal if $\gamma(\mathcal{G})=0$ or 1 , respectively. As a consequence of our results, we show that the commuting graphs of noncommutative rings of order $p^{2}$ and $p^{3}$ with unity are integral but not toroidal.

For any element $r$ of a ring $R$, its centralizer $C_{R}(r)$ is the set $\{s \in R: r s=$ $s r\}$. Let $\operatorname{Cent}(R)=\left\{C_{R}(r): r \in R\right\}$. Then $|\operatorname{Cent}(R)|$ gives the number of distinct centralizers in $R$. If $|\operatorname{Cent}(R)|=n$, then $R$ is called an $n$-centralizer ring. Recently, Dutta, Basnet, and Nath (see [10,9]) have characterized $n$-centralizer finite rings for $n=4,5,6,7$. In Section 3 , we show that the commuting graphs of $n$-centralizer finite rings are integral but not toroidal for $n=4,5$. Further, if $|R|=p^{n}$, where $p$ is a prime and $n$ is a positive integer, and $R$ is a $(p+2)$-centralizer ring, then we show that the commuting graph of $R$ is integral. We conclude this paper by computing the spectrum and genus of the commuting graphs of finite rings with some specific commuting probability. Recall that, the commuting probability $\operatorname{Pr}(R)$ of a ring $R$ is the "probability that a randomly chosen pair of elements of $R$ commute" (see $[16,7])$.

\section{Main results}

Recall that a CC-ring is a non-commutative ring $R$ such that $C_{R}(r)$ is commutative for all $r \in R \backslash Z(R)$. In [13], Erfanian et al. have initiated the study of CC-rings. In particular, they have computed the diameter of $\Gamma_{R}^{c}$ and showed that the clique number and chromatic number of $\Gamma_{R}^{c}$ are the same for a finite CC-ring $R$, where $\Gamma_{R}^{c}$ denotes the complement of $\Gamma_{R}$. The 
following theorem characterizes $\Gamma_{R}$ as disjoint unions of complete graphs if $R$ is a finite CC-ring.

Theorem 2.1. If $R$ is a finite $C C$-ring with distinct centralizers $S_{1}$, $S_{2}, \ldots, S_{n}$ of non-central elements of $R$, then

$$
\Gamma_{R}=\stackrel{i=1}{\sqcup}_{i=1}^{n} K_{\left|S_{i}\right|-|Z(R)|}
$$

Proof. Let $R$ be a finite CC-ring with distinct centralizers $S_{1}, S_{2}, \ldots, S_{n}$ of non-central elements of $R$. Let $S_{i}=C_{R}\left(s_{i}\right)$, where $s_{i} \in R \backslash Z(R)$ for $i=1,2, \ldots, n$. Let $s \in\left(S_{i} \cap S_{j}\right) \backslash Z(R)$ for some $i, j$ such that $1 \leq i \neq j \leq n$. Then $s$ commutes with $s_{i}$ as well as $s_{j}$. If $t \in C_{R}(s)$, then $t s_{i}=s_{i} t$ since $s_{i} \in C_{R}(s)$ and $R$ is a CC-ring. Therefore, $t \in C_{R}\left(s_{i}\right)$ and so $C_{R}(s) \subseteq C_{R}\left(s_{i}\right)$. Similarly we can show that $C_{R}\left(s_{i}\right) \subseteq C_{R}(s)$. Thus $C_{R}(s)=C_{R}\left(s_{i}\right)$. Also, it can be seen that $C_{R}(s)=C_{R}\left(s_{j}\right)$. Hence $C_{R}(s)=C_{R}\left(s_{i}\right)=C_{R}\left(s_{j}\right)$, which is a contradiction. Therefore, $S_{i} \cap S_{j}=Z(R)$ for $1 \leq i<j \leq n$. This shows that (2.1) holds.

Now, using Theorem 2.1, (1.1), and (1.2), we get the following corollary.

Corollary 2.2. If $R$ is a finite $C$ C-ring with distinct centralizers $S_{1}$, $S_{2}, \ldots, S_{n}$ of non-central elements of $R$, then

$$
\begin{aligned}
\operatorname{Spec}\left(\Gamma_{R}\right)=\left\{(-1)^{\sum_{i=1}^{n}\left|S_{i}\right|-n(|Z(R)|+1)},\left(\left|S_{1}\right|-|Z(R)|-1\right)^{1}\right. \\
\left.\ldots,\left(\left|S_{n}\right|-|Z(R)|-1\right)^{1}\right\}
\end{aligned}
$$

and

$$
\gamma\left(\Gamma_{R}\right)=\sum_{i=1}^{n} \gamma\left(K_{\left|S_{i}\right|-|Z(R)|}\right) .
$$

Corollary 2.3. Let $A$ be any finite commutative ring and $R$ be a finite CC-ring. Then

$$
\begin{aligned}
\operatorname{Spec}\left(\Gamma_{R \times A}\right)=\{ & (-1)^{\sum_{i=1}^{n}|A|\left(\left|S_{i}\right|-|Z(R)|\right)-n},\left(|A|\left(\left|S_{1}\right|-|Z(R)|\right)-1\right)^{1}, \\
& \left.\ldots,\left(|A|\left(\left|S_{n}\right|-|Z(R)|\right)-1\right)^{1}\right\}
\end{aligned}
$$

and

$$
\gamma\left(\Gamma_{R \times A}\right)=\sum_{i=1}^{n} \gamma\left(K_{|A|\left(\left|S_{i}\right|-|Z(R)|\right)}\right),
$$

where $\operatorname{Cent}(R)=\left\{R, S_{1}, \ldots, S_{n}\right\}$.

Proof. Let $R$ be a finite CC-ring and $\operatorname{Cent}(R)=\left\{R, S_{1}, \ldots, S_{n}\right\}$. Then $\operatorname{Cent}(R \times A)=\left\{R \times A, S_{1} \times A, \ldots, S_{n} \times A\right\}$. Hence, $R \times A$ is a CC-ring and the result follows from Corollary 2.2 noting that $Z(R \times A)=Z(R) \times A$. 
It seems to be difficult to determine all the finite non-commutative rings $R$ such that $\Gamma_{R}$ is integral. However, by Corollary 2.2 , it follows that $\Gamma_{R}$ is integral if $R$ is a finite CC-ring. Further, if $R$ is a finite CC-ring and $A$ is any finite commutative ring then, by Corollary $2.3, \Gamma_{R \times A}$ is also integral. In the next two results, we consider a particular class of CC-rings and compute the spectrum and genus of its commuting graph.

Theorem 2.4. If $p$ is a prime and the additive quotient group $\frac{R}{Z(R)}$ of a finite ring $R$ is isomorphic to $\mathbb{Z}_{p} \times \mathbb{Z}_{p}$, then

$$
\Gamma_{R}=\stackrel{\sqcup}{j=1}^{++1} K_{(p-1)|Z(R)|} .
$$

Proof. Let $a, b \in R$ such that

$$
\frac{R}{Z(R)}=\langle a+Z(R), b+Z(R)\rangle .
$$

Then it is clear that $p a, p b \in Z(R)$. Also, $a b \neq b a$ since $R$ is non-commutative. If $z \in Z(R)$, then we have

$$
\begin{aligned}
C_{R}(a) & =C_{R}(m a+z) \\
& =Z(R) \sqcup a+Z(R) \sqcup \cdots \sqcup(p-1) a+Z(R) \text { for } 1 \leq m \leq p-1
\end{aligned}
$$

and, for $1 \leq i \leq p$,

$$
\begin{aligned}
C_{R}(i a+b) & =C_{R}(i a+b+z) \\
& =Z(R) \sqcup(i a+b)+Z(R) \sqcup \cdots \sqcup((p-1) i a+(p-1) b)+Z(R) .
\end{aligned}
$$

Note that $C_{R}(x)=C_{R}(a)$ or $C_{R}(i a+b)$, where $1 \leq i \leq p$, for any $x \in R \backslash Z(R)$ and hence it is a commutative subring of $R$. Thus $R$ is a CC-ring. Therefore, by Theorem 2.1 we have

$$
\Gamma_{R}=K_{\left|C_{R}(a)\right|-|Z(R)|} \sqcup\left(\stackrel{i}{i=1}_{\sqcup} K_{\left|C_{R}(i a+b)\right|-|Z(R)|}\right) .
$$

That is,

$$
\Gamma_{R}=K_{(p-1)|Z(R)|} \sqcup\left(\stackrel{p}{i=1}_{\sqcup} K_{(p-1)|Z(R)|}\right)=\stackrel{p+1}{\sqcup} K_{i=1} K_{(p-1)|Z(R)|},
$$

since $\left|C_{R}(i a+b)\right|=p|Z(R)|=\left|C_{R}(a)\right|$ for $1 \leq i \leq p$.

Using Theorem 2.4, (1.1), and (1.2), we get the following corollary.

Corollary 2.5. If $p$ is a prime and the additive quotient group $\frac{R}{Z(R)}$ of a finite ring $R$ is isomorphic to $\mathbb{Z}_{p} \times \mathbb{Z}_{p}$, then

$$
\operatorname{Spec}\left(\Gamma_{R}\right)=\left\{(-1)^{\left(p^{2}-1\right)|Z(R)|-p-1},((p-1)|Z(R)|-1)^{p+1}\right\}
$$

and

$$
\gamma\left(\Gamma_{R}\right)=(p+1) \gamma\left(K_{(p-1)|Z(R)|}\right)
$$




\section{Some consequences}

In this section, we obtain several consequences of the results obtained in Section 2. It is not easy to determine all finite non-commutative rings such that their commuting graphs are planar or toridal. In this section, we characterize some finite rings whose commuting graphs are planar. We begin with the following result.

Proposition 3.1. Let $R$ be a finite ring such that the additive quotient group $\frac{R}{Z(R)}$ is isomorphic to $\mathbb{Z}_{p} \times \mathbb{Z}_{p}$, where $p$ is a prime. Then the following statements are true.

(a) $\Gamma_{R}$ is integral but not toroidal.

(b) $\Gamma_{R}$ is planar if and only if $p=2$ and $|Z(R)|=1,2,3$ or 4 , or $p=3$ and $|Z(R)|=1$ or 2 .

Proof. Part (a) follows from Corollary 2.5. If $p=2$, then $\gamma\left(\Gamma_{R}\right)=0$ if and only if $3 \gamma\left(K_{|Z(R)|}\right)=0$, which holds if and only if $|Z(R)|=1,2,3$ or 4 . If $p=3$, then $\gamma\left(\Gamma_{R}\right)=0$ if and only if $4 \gamma\left(K_{2|Z(R)|}\right)=0$, i.e., if and only if $|Z(R)|=1$ or 2 . Hence, part (b) follows.

Proposition 3.2. Let $R$ be a non-commutative ring of order $p^{2}$ for any prime $p$. Then the following is true.

(a) $\Gamma_{R}$ is integral but not toroidal.

(b) $\Gamma_{R}$ is planar if and only if $p=2,3$ or 5 .

Proof. Note that $|Z(R)|=1$ and the additive quotient group $\frac{R}{Z(R)}$ is isomorphic to $\mathbb{Z}_{p} \times \mathbb{Z}_{p}$. So, by Corollary 2.5 , we have

$$
\operatorname{Spec}\left(\Gamma_{R}\right)=\left\{(-1)^{p^{2}-p-2},(p-2)^{p+1}\right\}
$$

and

$$
\gamma\left(\Gamma_{R}\right)=(p+1) \gamma\left(K_{p-1}\right) .
$$

Thus it follows that $\Gamma_{R}$ is integral. Also, $\gamma\left(\Gamma_{R}\right) \neq 1$, that is, $\Gamma_{R}$ is not toroidal. Part (b) follows from the fact that $\gamma\left(\Gamma_{R}\right)=0$ if and only if $p=2,3$ or 5 .

Proposition 3.3. Let $R$ be a non-commutative ring with unity of order $p^{3}$ for any prime $p$. Then the following statements hold.

(a) $\Gamma_{R}$ is integral but not toroidal.

(b) $\Gamma_{R}$ is planar if and only if $p=2$.

Proof. Note that $|Z(R)|=p$ and the additive quotient group $\frac{R}{Z(R)}$ is isomorphic to $\mathbb{Z}_{p} \times \mathbb{Z}_{p}$. So, by Corollary 2.5 , we have

$$
\operatorname{Spec}\left(\Gamma_{R}\right)=\left\{(-1)^{p^{3}-2 p-1},\left(p^{2}-p-1\right)^{p+1}\right\}
$$


and

$$
\gamma\left(\Gamma_{R}\right)=(p+1) \gamma\left(K_{p^{2}-p}\right) .
$$

Thus $\Gamma_{R}$ is integral. Also, $\gamma\left(\Gamma_{R}\right) \neq 1$, that is, $\Gamma_{R}$ is not toroidal. Part (b) follows from the fact that $\gamma\left(\Gamma_{R}\right)=0$ if and only if $p=2$.

Proposition 3.4. If $R$ is a finite 4-centralizer ring, then $\Gamma_{R}$ is integral but not toroidal. Also $\Gamma_{R}$ is planar if and only if $|Z(R)|=1,2,3$ or 4 .

Proof. If $R$ is a finite 4-centralizer ring, then by [10, Theorem 3.2] we have that the additive quotient group $\frac{R}{Z(R)}$ is isomorphic to $\mathbb{Z}_{2} \times \mathbb{Z}_{2}$. Now the results follow from Proposition 3.1.

Proposition 3.5. If $R$ is a finite 5-centralizer ring, then $\Gamma_{R}$ is integral but not toroidal. Also $\Gamma_{R}$ is planar if and only if $|Z(R)|=1$ or 2 .

Proof. Let $R$ be a 5 -centralizer finite ring. Then by [10, Theorem 4.3] it follows that the additive quotient group $\frac{R}{Z(R)}$ is isomorphic to $\mathbb{Z}_{3} \times \mathbb{Z}_{3}$. Hence, the result follows from Proposition 3.1.

In the following proposition, we compute the spectrum and genus of a $(p+2)$-centralizer ring having order $p^{n}$ for any prime $p$ and positive integer $n$.

Proposition 3.6. Let $R$ be a ring of order $p^{n}$, where $p$ is a prime and $n$ is a positive integer. If $R$ is $(p+2)$-centralizer ring, then (2.2) and (2.3) are true.

Proof. It follows, by [10, Theorem 2.12], that the additive quotient group $\frac{R}{Z(R)}$ is isomorphic to $\mathbb{Z}_{p} \times \mathbb{Z}_{p}$. Therefore, using Corollary 2.5 we get the required result.

The commuting probability $\operatorname{Pr}(R)$ of a finite ring $R$ is given by the ratio

$$
\frac{|\{(r, s) \in R \times R: r s=s r\}|}{|R|^{2}} .
$$

The study of $\operatorname{Pr}(R)$ was initiated by MacHale [16] in 1976. MacHale [16] proved the following result.

Theorem 3.7. Let $R$ be a finite ring and $p$ the smallest prime dividing $|R|$. Then

$$
\operatorname{Pr}(R) \leq \frac{p^{2}+p-1}{p^{3}}
$$

The equality holds if and only if the additive quotient group $\frac{R}{Z(R)}$ is isomorphic to $\mathbb{Z}_{p} \times \mathbb{Z}_{p}$.

We conclude this paper with the following two consequences of Corollary 2.5 and Theorem 3.7. 
Proposition 3.8. Let $R$ be a finite ring with $\operatorname{Pr}(R)=\frac{5}{8}$. Then

$$
\operatorname{Spec}\left(\Gamma_{R}\right)=\left\{(-1)^{3(|Z(R)|-1)},(|Z(R)|-1)^{3}\right\}
$$

and

$$
\gamma\left(\Gamma_{R}\right)=3 \gamma\left(K_{|Z(R)|}\right) .
$$

Proposition 3.9. Let $R$ be a finite ring and $p$ the smallest prime dividing $|R|$. If (3.1) holds, then (2.2) and (2.3) are satisfied.

\section{Acknowledgments}

The authors would like to thank the referees for their valuable comments and suggestions.

\section{References}

[1] A. Abdollahi, Commuting graphs of full matrix rings over finite fields, Linear Algebra Appl. 428 (2008), 2947-2954.

[2] A. Abdollahi and E. Vatandoost, Which Cayley graphs are integral, Electron. J. Combin. 16 (2009), R122, 17 pages.

[3] M. Afkhami, Z. Barati, N. Hoseini, and K. Khashyarmanesh, A generalization of commuting graphs, Discrete Math. Algorithm. Appl. 7 (2015), 1450068, 11 pages.

[4] S. Akbari, M. Ghandehari, M. Hadian, and A. Mohammadian, On commuting graphs of semisimple rings, Linear Algebra Appl. 390 (2004), 345-355.

[5] S. Akbari and P. Raja, Commuting graphs of some subsets in simple rings, Linear Algebra Appl. 416 (2006), 1038-1047.

[6] J. Battle, F. Harary, Y. Kodama, and J. W. T. Youngs, Additivity of the genus of a graph, Bull. Amer. Math. Soc. 68 (1962), 565-568.

[7] J. Dutta, D. K. Basnet, and R. K. Nath, On commuting probability of finite rings, Indag. Math. (N. S.) 28 (2017), 372-382.

[8] J. Dutta, D. K. Basnet, and R. K. Nath, On generalized non-commuting graph of a finite ring, Algebra Colloq. 25 (2018), 149-160.

[9] J. Dutta, D. K. Basnet, and R. K. Nath, A note on n-centralizer finite rings, An. Ştiinţ. Univ. Al. I. Cuza Iaşi. Mat. 64(1) (2018), 161-171.

[10] J. Dutta, D. K. Basnet, and R. K. Nath, Characterizing some rings of finite order, submitted for publication, available at arXiv:1510.08207.

[11] J. Dutta and R. K. Nath, Finite groups whose commuting graphs are integral, Mat. Vesnik 69 (2017), 226-230.

[12] J. Dutta and R. K. Nath, Spectrum of commuting graphs of some classes of finite groups, Matematika 33 (2017), 87-95.

[13] A. Erfanian, K. Khashyarmanesh, and Kh. Nafar, Non-commuting graphs of rings, Discrete Math. Algorithm. Appl. DOI: 10.1142/S1793830915500275.

[14] F. Harary and A. J. Schwenk, Which graphs have integral spectra?, in: Graphs and Combinatorics, Lect. Notes Math. Vol 406, Springer-Verlag, Berlin, 1974, pp. 45-51.

[15] G. Indulal and A. Vijayakumar, Some new integral graphs, Appl. Anal. Discrete Math. 1 (2007), 420-426.

[16] D. MacHale, Commutativity in finite rings, Amer. Math. Monthly 83 (1976), 30-32.

[17] A. Mohammadian, On commuting graphs of finite matrix rings, Comm. Algebra 38 (2010), 988-994. 
[18] G. R. Omidi and E. Vatandoost, On the commuting graph of rings, J. Algebra Appl. 10 (2011), 521-527.

[19] E. Vatandoost, F. Ramezani, and A. Bahraini, On the commuting graph of noncommutative rings of order $p^{n} q$, J. Linear Topol. Algebra 3 (2014), 1-6.

[20] L. Wang, X. Li, and C. Hoede, Two classes of integral regular graphs, Ars Combin. 76 (2005), 303-319.

[21] A. T. White, Graphs, Groups and Surfaces, North-Holland Mathematics Studies 8, American Elsevier Publishing Co., New York, 1973.

Department of Mathematical Sciences, Tezpur University, Napaam-784028, Sonitpur, Assam, India

E-mail address: jutirekhadutta@yahoo.com

E-mail address: w.n.fasfous@gmail.com

E-mail address: rajatkantinath@yahoo.com 